\title{
Cases and dialectical arguments An approach to case-based reasoning
}

\author{
Bram Roth $^{1}$ and Bart Verheij ${ }^{2}$ \\ ${ }^{1}$ Universiteit Maastricht, \\ BramRothecs.com \\ ${ }^{2}$ Rijksuniversiteit Groningen
}

\begin{abstract}
Case-based reasoning in the law is a reasoning strategy in which legal conclusions are supported by decisions made by judges. If the case at hand is analogous to a settled case, then by judicial authority one can argue that the settled case should be followed. Case-based reasoning is a topic where ontology meets logic since one's conception of cases determines one's conception of reasoning with cases. In the paper, it is shown how reasoning with cases can be modelled by comparing the corresponding dialectical arguments. A unique characteristic thereby is the explicit recognition that it is in principle contingent which case features are relevant for case comparison. This contigency gives rise to some typical reasoning patterns. The present work is compared to other existing approaches to reasoning by case comparison, and some work on legal ontologies is briefly discussed regarding the role attributed to cases.
\end{abstract}

\section{Introduction}

Case-based reasoning is a common type of argumentation in the law, in which legal conclusions are supported with decided cases. If some decided case is sufficiently similar to the case at hand, then under the doctrine of stare decisis one should not depart from that decision, and the same conclusion should hold.

The principle of stare decisis holds that in settling new cases judges tend to adhere to prior decisions, in the sense that once a question of law is decided, the decision is normally not departed from afterwards. There are a number of reasons to adhere to earlier decisions. First, courts are often formally bound by decisions made by courts higher up in the hierarchy and by their own decisions (cf. Cross 1977, pp. 103f.). Second, a departure from settled cases would make judicial decisions uncertain and unpredictable, so that one could never be sure about the status of one's legal claims in court. Third, it is fair and just to treat all individuals equally under the law, and to make no difference between people in similar cases. Fourth, adhering to decisions turns out to be an economic and efficient practice (Bankowski 1997, p. 490). 
Different methods to adhere to decisions have been described in the literature (Llewellyn 1960, pp. 77-84), and two of them will be discussed next: rule extraction and case comparison.

In the first method the reasons underlying the conclusion are isolated, the reasons and conclusion are generalised (or 'universalised', cf. Hage 1997, p. 47; MacCormick 1987, pp. 162f.) into a rule that can explain the outcome. This mechanism is called the 'rule extraction method' for short.

The second method of adhering to a decision is by assigning the corresponding legal conclusion to the new case as an authoritative example (cf. Ashley 1990, pp. 1112; Aleven 1997, pp. 58; Oliphant 1928, pp. 144f.). If the decided case is 'sufficiently similar' to the case at hand, then one can argue by analogy and judicial authority that the decided case should be followed and that the same decision should be taken once more.

The methods of rule extraction and case comparison can be presented schematically as a list of reasoning steps, as in Figure 1. The figure illustrates that there are strong relations between both methods, in the sense that they involve comparable reasoning steps.

\begin{tabular}{|c|c|}
\hline Rule extraction method & Case comparison method \\
\hline $\begin{array}{l}\text { (1) Extracting rules from decided } \\
\text { cases } \\
\text { (2) Showing that rule conditions are } \\
\text { satisfied }\end{array}$ & $\begin{array}{l}\text { (1) Selecting relevant case facts } \\
\text { (2) Establishing an analogy between } \\
\text { cases }\end{array}$ \\
\hline $\begin{array}{ll}\text { (3a) Applying } & \text { (3b) Pointing } \\
\text { extracted rules } & \text { out exceptions to } \\
\text { to the case at } & \text { extracted rules } \\
\text { hand } & \end{array}$ & \begin{aligned} (3a) Following & \multicolumn{1}{c}{$(3 \mathrm{~b})$} \\
decided cases in & Distinguishing \\
the case at hand & $\begin{array}{l}\text { decided cases } \\
\text { from the case at } \\
\text { hand }\end{array}\end{aligned}$ \\
\hline
\end{tabular}

Figure 1: the methods of rule extraction and case comparison

Our discussion of the two methods to adhere to decisions show that case-based reasoning is a topic where ontology meets logic. The two methods correspond to different ontological conceptions of cases that determine different logical analyses of reasoning. When cases are considered as authoritative sources of rules (as in the rule extraction method), reasoning with cases only differs from rule-based reasoning in the phase of rule extraction. After that phase, the extracted rules are applied, just like other rules. From an ontological point of view, the rule extraction method treats cases basically as sets of rules. In the method of case comparison, cases are considered differently, namely as authoritative sources of arguments and decisions. Cases are treated as wholes during all phases of reasoning. Ontologically, the case comparison method views cases basically as sets of arguments and decisions. 
In the present paper, we have chosen the case comparison method as the basis for modelling case-based reasoning. The model is informally presented. Roth (2003) gives further details and a formal elaboration.

\section{Dialectical arguments}

To arrive at a systematic analysis of cases, it is convenient to have a graphical representation of the argumentation in the cases. To this end tree-like structures are introduced, called dialectical arguments; cf. Verheij's (2000; 2001; 2003) naïve dialectical arguments and Loui's (1997) and Loui and Norman's (1995, p. 164) records of disputation. Dialectical arguments consist of statements that support or attack other statements, the support and attack relations being represented by arrows. Here is a simplified example for the domain of Dutch dismissal law.

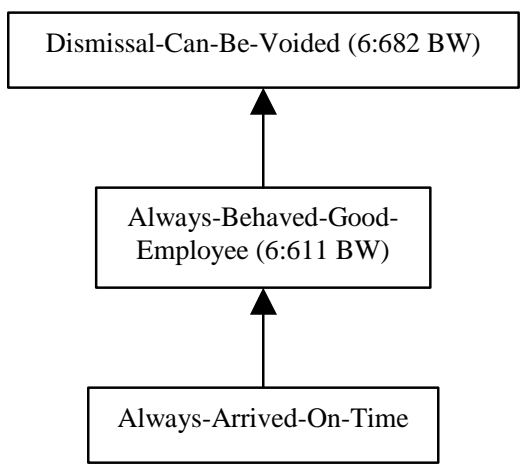

At the top of this figure one finds the legal conclusion that the dismissal can be voided, to which a judge can decide on the basis of article 6:682 of the Dutch Civil Code (art. 6:682 BW). The statement in the middle is that the dismissed person has always behaved like a good employee, a general obligation for employees that is codified in article 6:611 of the Dutch Civil Code (art. 6:611 BW). An arrow upwards indicates that the conclusion that the dismissal can be voided is supported by the statement that the dismissed person has always behaved like a good employee. This statement is in turn supported by the statement that the employee always arrived on time for work.

Conclusions cannot only be supported, but they can be attacked as well. Attacks are represented by arrows ending in a solid square. In the following figure one finds an example of this. 


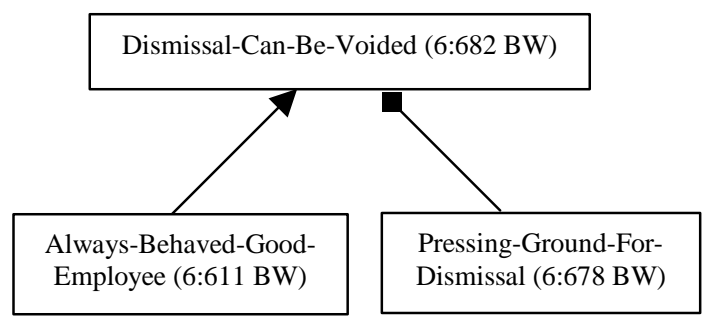

Here the conclusion that the dismissal can be voided is attacked by the statement that there is a pressing ground for dismissal according to article 6:678 of the Dutch Civil Code (art. 6:678 BW).

It is a key feature of the present approach that it can also be supported and attacked that a statement supports a conclusion (cf. Toulmin's warrants, 1958, pp. 98f.), or attacks it (Hage 1997, p. 166; Verheij 1996, pp. 200-201; Pollock 1995, pp. 41 and 86; Pollock 1987, p. 485).

A step forward to deal with this is to treat it as a statement itself that the conclusion is supported or attacked (cf. Verheij's DEFLoG, 2000, pp. 5f.1; 2003; see also Verheij 1999, pp. 45f.). Accordingly, one can represent by an arrow pointing at another arrow that it is supported or attacked that a statement supports or attacks a conclusion. This gives rise to a kind of entanglement of dialectical arguments (Roth 2001, pp. 31-33). An example is in the following figure.

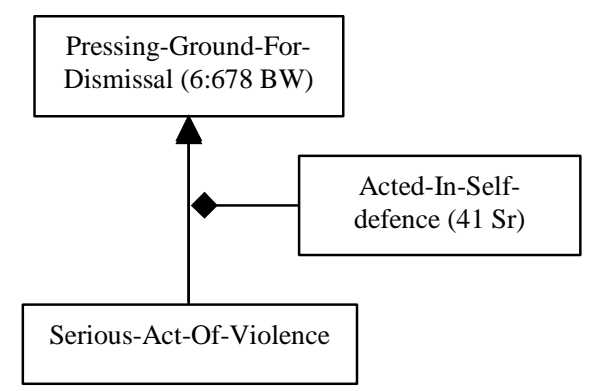

At the top of this figure one finds the conclusion that there is a pressing ground for dismissal according to article 6:678 of the Dutch Civil Code. The conclusion is supported by the statement that the employee committed a serious act of violence. However, it is attacked that having committed a serious act of violence supports that there is a pressing ground for dismissal. The attacking statement is that the employee acted in self-defence, which is a general ground of justification according to article 41 of the Dutch Penal Code (art. $41 \mathrm{Sr}$ ). As a result, on the sole ground that the employee committed a serious act of violence, the conclusion does not follow that there is a pressing ground for dismissal.

It can also be supported that a statement supports or attacks a conclusion. An example is in the following figure. 


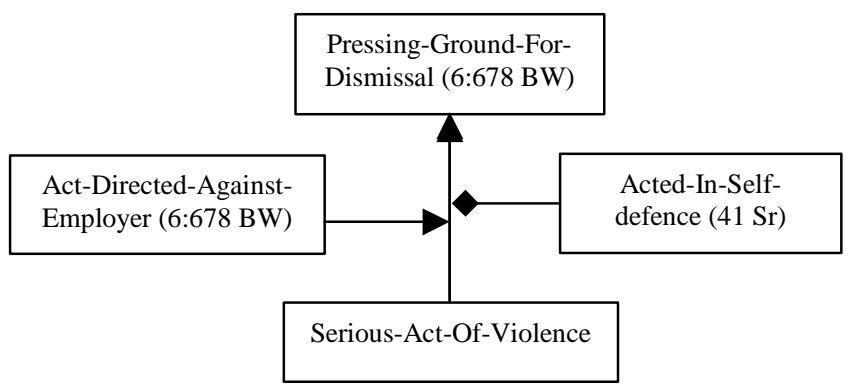

Here the statement is made that the violent act was directed against the employer. This statement supports that having committed a serious act of violence supports that there is a pressing ground for dismissal, in accordance with article 6:678 of the Dutch Civil Code (art. 6:678 BW).

Dialectical arguments will be used as a convenient graphical representation of the argumentation in cases, but the question which conclusions follow is not answered by interpreting dialectical arguments regarding the status of the statements involved (cf. Verheij's dialectical interpretations, 2000, 2003).

\section{Modelling case-based reasoning by case comparison}

In this section reasoning by case comparison is modelled as a variant of reasoning a fortiori that involves dialectical arguments. The relevant case features appearing in these dialectical arguments are given by what is called a comparison basis. It is explained under which conditions a - possibly contradictory - set of settled cases can help decide a problem case.

\subsection{Case comparison}

It is the purpose of case comparison to determine whether a settled case can be followed in a problem case. Intuitively one can certainly follow a settled case where, if there is at least as much support for the conclusion in the problem case. Then, by a kind of reasoning a fortiori, the conclusion should hold again.

The support for a conclusion is determined by the dialectical argument for it. In this connection we will use the term dialectical support for a conclusion. Case comparison will come down to comparing dialectical arguments regarding the dialectical support for their conclusion.

In the following a number of examples of increasing complexity are given. 
Settled case

$c$ : Dismissal-Can-Be-Voided

$a$ : Always-Behaved-Good-Employee

$b$ : Serious-Act-Of-Violence

$d$ : Working-Atmosphere-Not-Affected

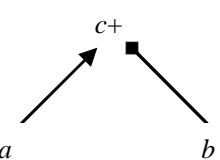

Problem case

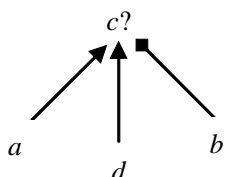

In this figure there is a settled case on the left where the conclusion $(c)$ was drawn that a person's dismissal could be voided, as indicated by the plus sign. On the right there is a problem case where this conclusion is an issue, as indicated by the question mark.

In both cases the conclusion $(c)$ that the dismissal can be voided is supported by the statement $(a)$ that the person has always behaved like a good employee. The conclusion $c$ is attacked by the statement $(b)$ that the employee committed a serious act of violence. In the problem case the conclusion $c$ is also supported by the statement $(d)$ that the working atmosphere has not been affected by the dismissal. As a result, there is more dialectical support for $c$ in the problem case, so that it should follow there as well.

Another example is in the following figure.

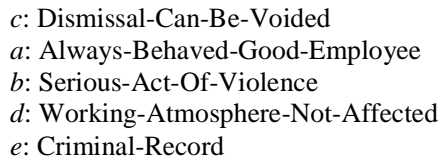

In the settled case the conclusion $c$ is attacked by the statement $(e)$ that the employee has a criminal record. Together with the difference $d$ already discussed, this means that there is more dialectical support for $c$ in the problem case. As a result, the conclusion $(c)$ that the dismissal can be voided holds again.

Dialectical arguments can have a more complex structure. An example is in the following figure.

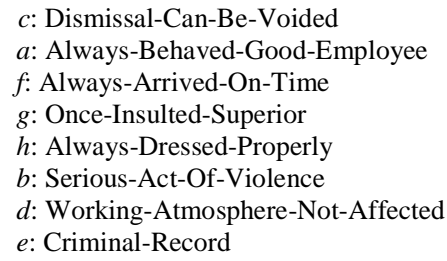

\section{Problem case}

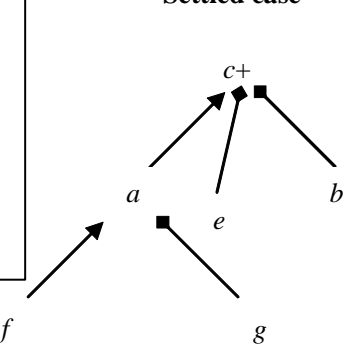

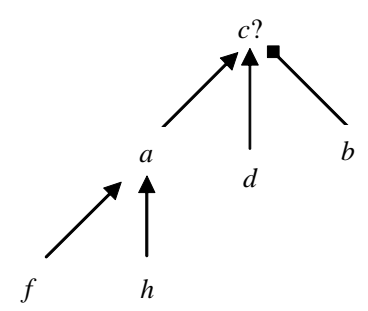


In this situation there is more dialectical support for the statement $a$ in the problem case. In accordance with this, there is more dialectical support for conclusion $c$ in the problem case than in the settled case, so that the conclusion can follow in the problem case as well. Note that for concluding to the outcome that there is more dialectical support for $c$ in the problem case, it does not matter how the conflict with regard to the intermediate $a$ is to be resolved.

It can itself be supported or attacked that one statement supports or attacks another. An example is in the following figure.
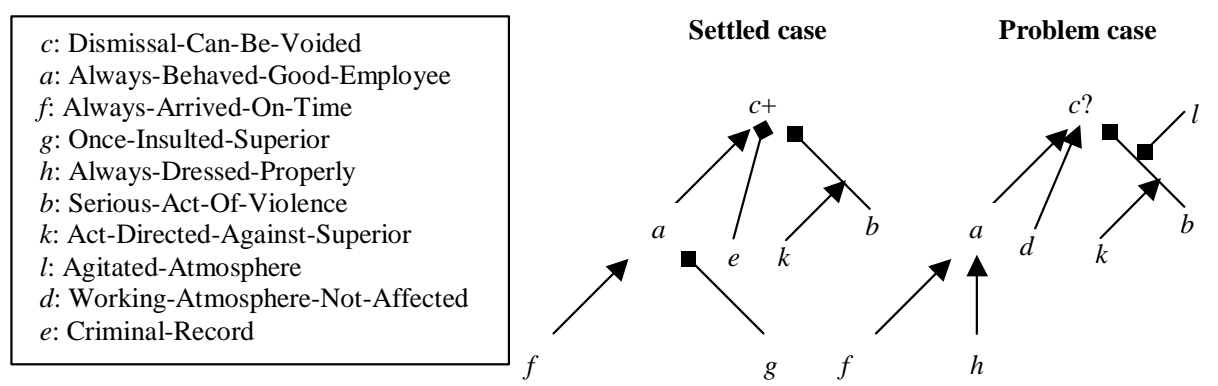

In this situation there is less dialectical support for the statement that $b$ attacks $c$ in the problem case, due to the attack by the statement $(l)$ that the violent act took place in an agitated atmosphere. As a consequence, the problem case provides more dialectical support for conclusion $c$ than the settled case, so that the conclusion can follow in the problem case as well. Note that for concluding to this result it is not necessary to resolve the conflict with regard to the attack by $b$.

\subsection{The comparison basis}

When comparing dialectical arguments one finds different types of statement. The conclusion at the top of these dialectical arguments tends to be the statement of an abstract legal state of affairs. At the bottom of the arguments one normally finds statements of non-legal and concrete facts.

Not all these states of affairs are relevant for the purpose of case comparison. The mere fact that John smashed a window, for example, can be irrelevant for the purpose of comparing his case with another case where liability for damage is an issue. At this point a distinction is therefore postulated between relevant and irrelevant states of affairs. From this point onwards all statements corresponding to relevant states of affairs will be called factors, to stress their role in case comparison. ${ }^{1}$

In general it is disputable which statements are factors and which are not. In other words, in the law it depends on a contingent choice which factors are taken into account when comparing cases. Among other things this choice will depend on the legal domain under consideration, such as dismissal or trade secret law.

${ }^{1}$ Cf. the 'factors' in HYPO and CATO (cf. Ashley 1990, pp. 37-38). 
Some factors have the intuitive property that what supports them can be ignored for the purpose of case comparison, and that for this purpose all that matters is whether or not they can be derived. By definition these factors form a set called the comparison basis, and the factors in this set are called basic. ${ }^{2}$

In the figure below the comparison basis is visualised by drawing a line of division through the dialectical arguments. Statements above the line correspond to relevant states of affairs that have to be considered for the purpose of case comparison, while states of affairs below the line can be ignored. If the comparison basis is chosen as in the figure, then the two cases are comparable regarding their dialectical support for the conclusion $(c)$ that the dismissal can be voided.

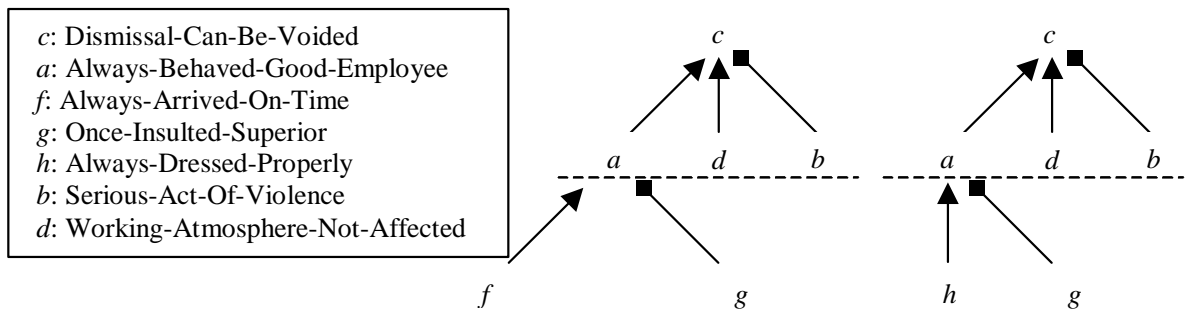

The following figure shows a different choice for the comparison basis in the same situation, according to which the supporting factors $f$ and $h$ are relevant as well. In other words, it makes a difference now whether the employee was always dressed properly, or always arrived on time. As a result, the two cases are now not comparable regarding the dialectical support for the conclusion.

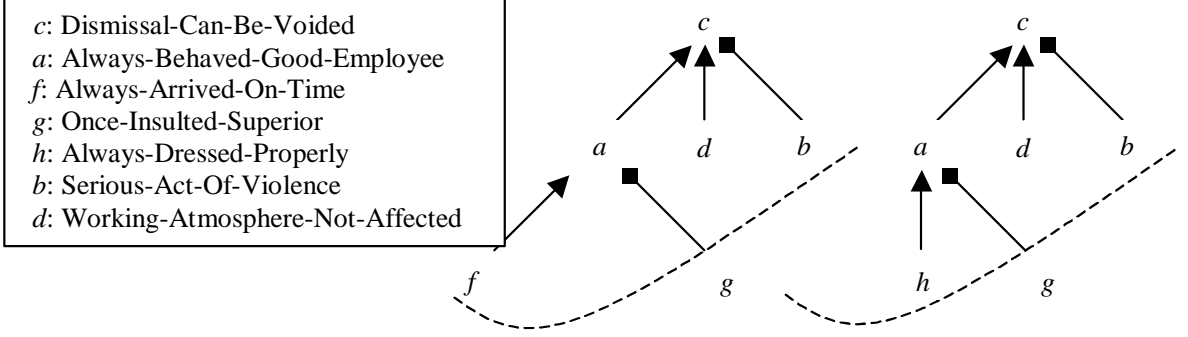

These figures show that the comparison outcomes depend on the particular division made between factors and non-factors. They also illustrate the point that it is disputable which statements will count as basic factors.

${ }^{2}$ Cf. the 'base-level factors' in CATO (Aleven 1997 pp. 23 and 47). 


\subsection{The entangled factor hierarchy}

Let us consider all factors that follow from the comparison. Together these factors make up a representation of the argumentation pertaining to some domain of law: an ontology for the domain under consideration. An example of such a representation is depicted in the figure below.

\section{Entangled factor hierarchy (part)}

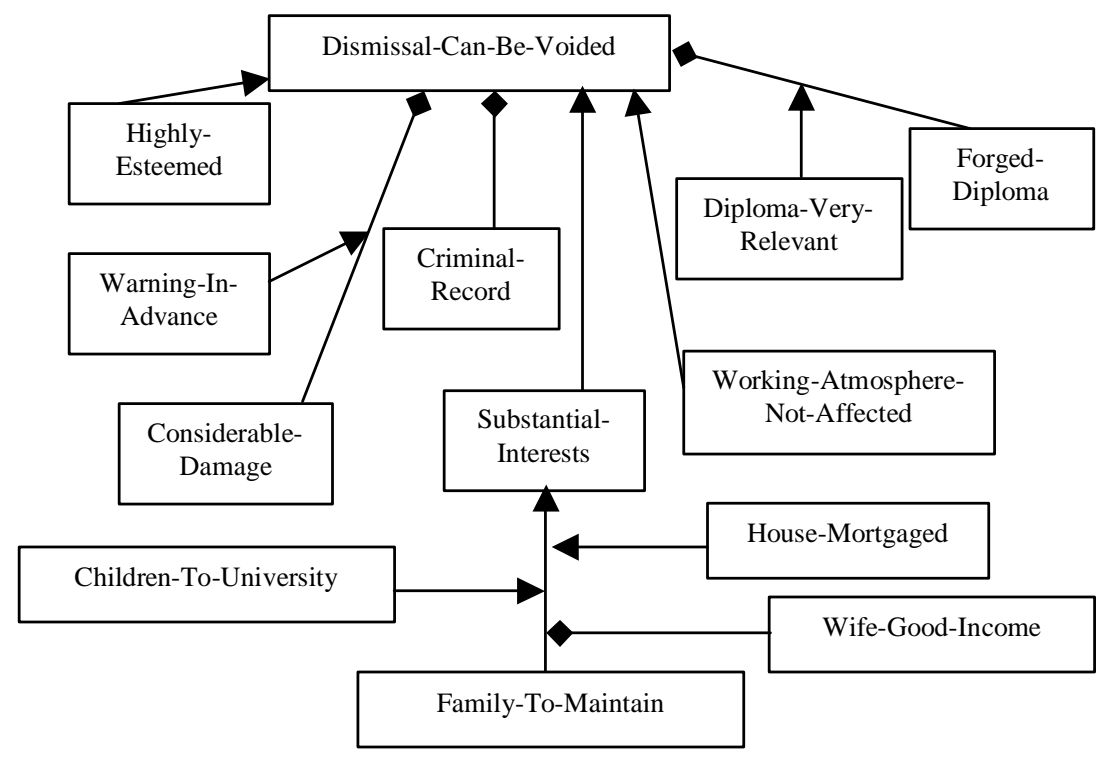

The representation thus obtained is similar to the so-called 'Factor Hierarchy' employed by CATO (Aleven 1997, pp. 44-45) as a case-independent knowledge structure. However, in contrast with CATO the present approach also allows for supporting and attacking statements of support or attack, which is why the present structure is called an entangled factor hierarchy (Roth 2001, pp. 31-33)

\subsection{Which settled cases are relevant?}

It is shown next how one can compare a number of cases regarding their dialectical support for some conclusion, and use the comparison to select the settled cases that are relevant to resolve a given problem case.

The settled cases are ordered regarding their dialectical support, which is represented graphically by placing the cases as dots on a line. 


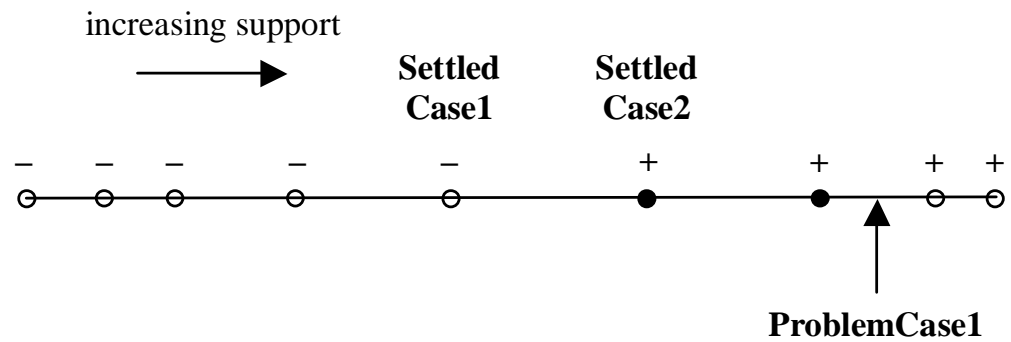

In this figure the small circles represent settled cases. The conclusion of each settled case is indicated by a plus or a minus sign, standing for a conclusion (say $c$ ) or its opposite conclusion $(\neg c)$, respectively. From left to right there is increasing dialectical support for the conclusion $(c)$.

ProblemCase1 is a problem case which has two positively decided cases on its left, represented by solid small circles. These are precedents for their conclusion $(c)$, because ProblemCase1 provides more dialectical support for the conclusion. Since there are no precedents for the opposite conclusion, it is clear that ProblemCase1 should be decided positively as well.

In exceptional situations a judge may decide a case without taking recourse to the stare decisis principle. A reason for this could be, for instance, that a judge wants to take into account a changing view on the law. Whatever the reasons to depart from stare decisis, if it is done the set of precedents can become contradictory, and then it can become problematic to draw conclusions in problem cases. An example is in the following figure.

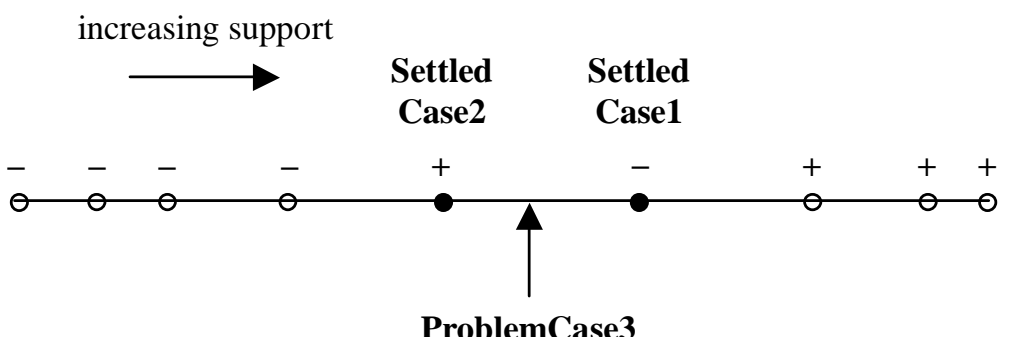

Obviously SettledCase1 is now to the right of SettledCase2. As a result, the two decisions are contradictory, in the sense that the case with more support was decided negatively, while in the case with less support a positive conclusion was drawn. In other words, for whatever reason, at least one of these cases must have been decided against the principle of stare decisis.

Now consider ProblemCase3, which is positioned between these two settled cases. Obviously, SettledCase1 is a precedent for the opposite conclusion $(\neg c)$ with respect to ProblemCase3, while SettledCase2 is a precedent for the conclusion itself $(c)$. As a result, the precedents cannot both be followed here. 
This example shows that if precedents have been decided in a contradictory way and against the principle of stare decisis, then sometimes no conclusion follows from them in a problem case.

\section{Applications}

The ideas presented in the foregoing can be applied to arrive at an account of interesting reasoning patterns in case comparison. It is possible to accommodate the well-known analogising and distinguishing moves (Ashley 1990, pp. 25f; Aleven 1997, pp. 58f.; Roth 2003, pp. 86). A number of arguments on the importance of distinctions and similarities can also be captured, viz. downplaying and emphasising (cf. Aleven 1997, pp. 62f.). As an example of the latter kind, consider the situation of the following figure. Here a settled case is cited for the conclusion $(c)$ that the dismissal can be voided.
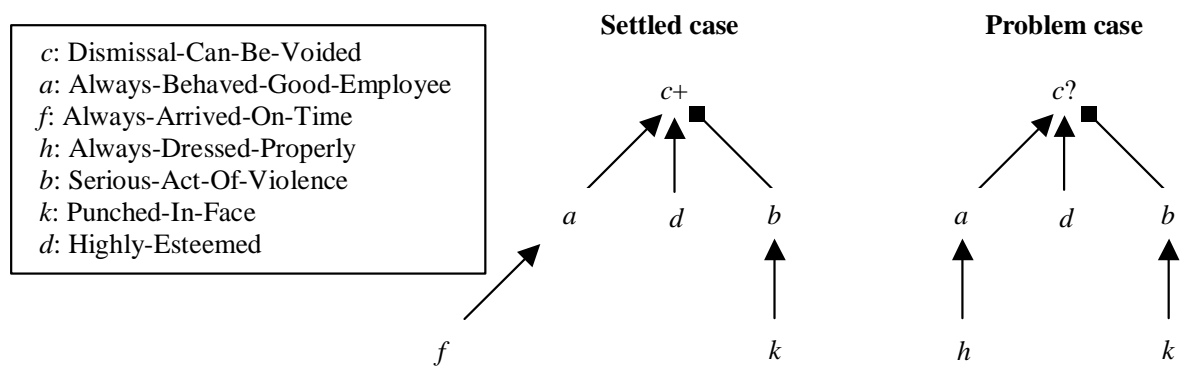

Suppose that the settled case is distinguished from the problem case by pointing out the significant distinction $(f)$ that the employee always arrived on time.

Downplaying with an abstract interpretation that is a significant similarity

This way of downplaying is done by replacing a distinction with an abstract interpretation of it. Then the cases are compared in terms of this abstract interpretation, which then is a significant similarity. See the following figure. 


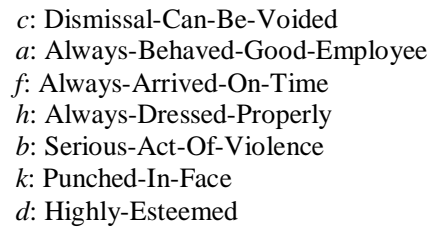

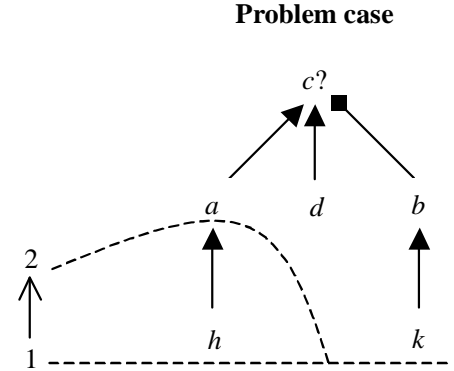

In this figure there are two comparison bases. One is indicated with a straight dashed line at level 1, a second is represented by a curved dashed line at level 2.

The figure shows that if the comparison basis at level 1 is chosen, then the factor $(f)$ that the dismissed employee always arrived on time is a significant distinction. The distinction applies to the settled case and not to the problem case, and it supports the more abstract factor $(a)$ that the person always behaved like a good employee.

The comparison can also be done in terms of this more abstract factor, which comes down to choosing another comparison basis. This second comparison basis differs from the first in that the original factor $f$ is replaced with its abstract interpretation $a$. In the figure this second comparison basis is indicated by the curved dashed line at level 2.

Relative to this second comparison basis the factor $f$ is no significant distinction because it is not a factor. Its abstract interpretation $a$ is a basic factor that applies to both cases. As a result, relative to the second comparison basis the factor $a$ is a significant similarity between the cases.

Emphasising with an abstract interpretation that is a significant distinction Emphasising a distinction also involves an abstract interpretation of the distinction. The difference is, though, that here the abstract interpretation is a significant distinction and not a significant similarity. See the following figure.

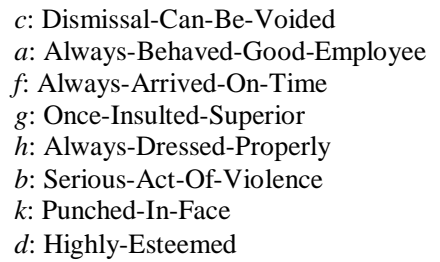

\section{Settled case}

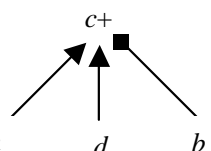

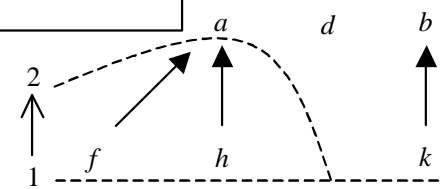

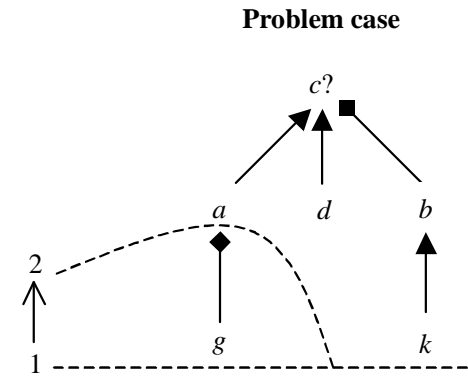


Again the focus is on the distinction $f$. The original distinction $f$ is replaced with its abstract interpretation $a$, and again this yields another comparison basis at level 2 .

This abstract interpretation $a$ is a basic factor that applies to the settled case but not to the problem case. As a result, relative to the second comparison basis the factor $a$ is a significant distinction between the cases.

These examples show how distinctions can be downplayed or emphasised. In a similar way one can downplay or emphasise significant similarities (Roth 2003, pp. 95f.).

\section{Related research}

In Section 5.1 we discuss research that specifically focuses on case-based reasoning in the law. Section 5.2 addresses research on legal ontologies.

\subsection{Case-based reasoning}

The HYPO system represents one of the most important contributions to case-based reasoning in the field of Artificial Intelligence and Law. HYPO is an implemented model of case-based reasoning that can generate realistic arguments with cases on the basis of expert background knowledge on supporting and attacking factors (p. 26). The background knowledge used for generating arguments is represented by tagging factors with a plus or a minus sign if they support a conclusion or its opposite, respectively.

In HYPO it is not disputable whether or not a factor supports some conclusion or its opposite, however. Recall that in approach adopted in this paper, in contrast, it can be supported or attacked that one statement supports or attacks another (entanglement).

The CATO program (Aleven 1997; Aleven 1996; Aleven and Ashley 1997) uses a model of case-based argumentation in an instructional setting, to teach students basic skills of arguing with cases.

The CATO model of case-based reasoning relies on a Factor Hierarchy, a body of case-independent background knowledge about how the relevant factors in some domain relate to each other. Among other things the Factor Hierarchy is used to reason about the significance of distinctions between cases (downplaying and emphasizing). To produce such arguments CATO can infer abstract interpretations of with the help of the links encoded in its Factor Hierarchy, thereby guided by special strategic heuristics. As shown previously, such downplaying and emphasising arguments can also be accommodated in the present approach. There presently are no heuristics to guide a strategic choice among several possible ways of downplaying or emphasising, however.

Another difference with the present approach is that in CATO it cannot be supported or attacked that a factor supports a conclusion or its opposite. 
Prakken and Sartor (1998) deal with case-based reasoning as a kind of dialectical argumentation. Cases are represented by collections of rules from which arguments can be constructed (p. 256). The conflict resolving potential of settled cases is exploited by interpreting them as sources of priority information on conflicting rules (p. 256).

In Prakken and Sartor's system the focus is on analogizing and distinguishing as ways of reasoning with cases. These two ways of reasoning are treated as a kind of premise introduction (p. 261) or theory construction (cf. Prakken 2000, pp. 51f.). They involve the introduction of new rules on the basis of rules of settled cases.

This way of reasoning with cases differs from the present approach in that a suitable comparison outcome must be established before a settled case can be followed. There must be at least as much dialectical support for a conclusion in the problem case, and only then can one draw the conclusion.

Prakken and Sartor want to deal with case comparison in terms of HYPO's criterion of on pointness (p. 267). Due to problems in connection with derivable factors, however, they choose not to define comparison outcomes in terms of on pointness (p. 270). Instead they simply assume that some criterion for on pointness has already been agreed upon, as part of the premises from which the debate starts (pp. 270-271).

This is in contrast with the present approach, where the outcomes of case comparison are defined in terms of the level of dialectical support for a conclusion.

A final difference with the present approach is that the set of factors relevant for case comparison is not introduced explicitly to acknowledge the contingency of this set.

Bench-Capon and Sartor (2001) have proposed an approach in which reasoning with cases is treated as the construction and use of theories.

In their model, theories are intended to explain decided cases in terms of the values promoted by the legal system as a whole, and to help predict the outcomes of new cases. Briefly, decisions are interpreted as evidence for rule priorities, which are in turn interpreted in terms of priorities between the values upheld by the rules. These value priorities can then be used to derive new rule priorities that can help decide problem cases.

Bench-Capon and Sartor's model does not recognise explicitly that in the law it depends on a contingent choice which factors are relevant for case comparison.

Hage gives an account of case-based reasoning in Reason-Based Logic (Hage 1997, Chapters IV and V; Verheij 1996, Chapters 2 and 3). Reason-Based Logic is a logical system which involves the use of rules and principles in legal reasoning, and which allows for exceptions to rules or principles, to the effect that conclusions cease to hold (Hage 1997, pp. 137-138, 141-143 and 150-151).

In Hage's reason-based account of case-based reasoning, case comparison is done in terms of the reasons for or against the disputed conclusion. In other words, Hage's account only captures one-step arguments. 
Another important difference between Hage's account and the present one is that while entanglement of factors can in principle be captured in Hage's Reason-Based Logic, it does not play a role in his account of case comparison.

Two ways of categorising approaches to case-based reasoning are illustrated next. First, one can distinguish the approaches by the method of employing cases that they focus on: the rule extraction or case comparison method. Second, one can categorise approaches by their relative emphasis on the legal conclusions that follow by comparison with settled cases, or the reasoning patterns along which they follow.

An example of a categorisation along these distinctions is in the following figure.

\section{Positioning approaches to case-based reasoning}

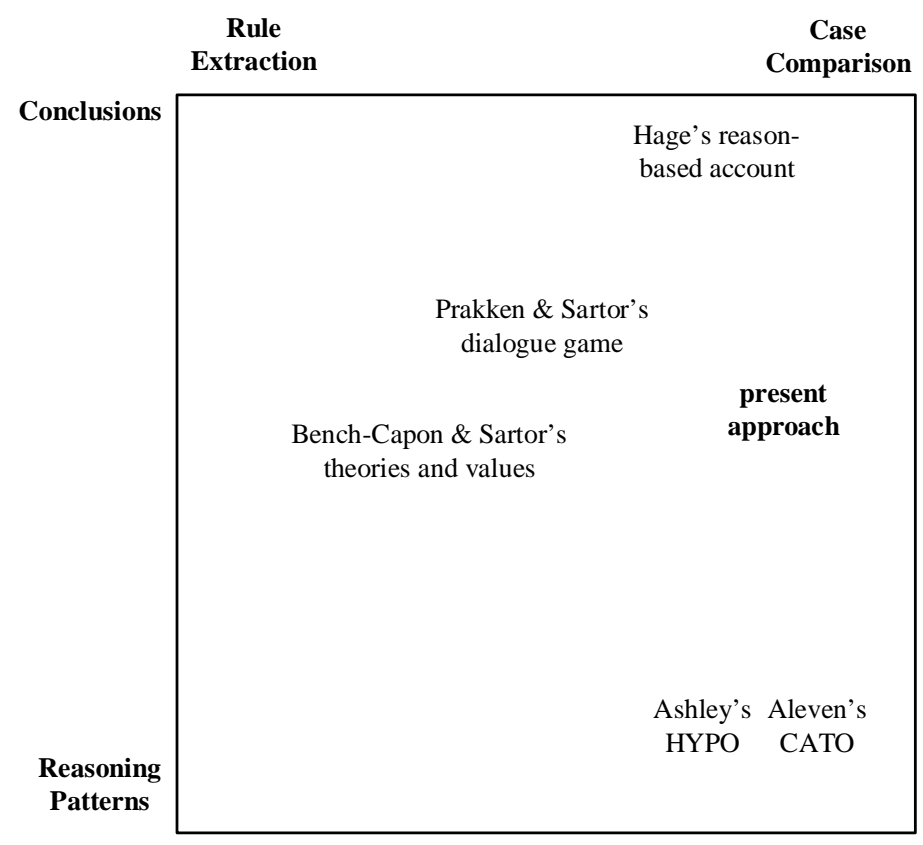

\subsection{Cases in legal ontologies}

There exists a lively research community dealing with the topic of legal ontologies from an artificial intelligence perspective (cf., e.g., Visser \& Winkels 1997, Winkels, Van Engers \& Bench-Capon 2001). ${ }^{3}$ We briefly discuss work on legal ontologies and

\footnotetext{
${ }^{3}$ A useful web site for pointers to research concerning legal ontologies is the home page of Radboud Winkels (http://www.lri.jur.uva.nl/ winkels/).
} 
address the question how cases are treated. It is also indicated whether the work is closer to the rule extraction method or to the case comparison method.

Valente (1995) has presented a functional ontology of the law. Valente's functional perspective on the law is reflected in the categories of legal knowledge he distinguishes: normative knowledge, world knowledge, responsibility knowledge, reactive knowledge, meta-legal knowledge and creative knowledge. Valente does not directly address case-based reasoning (although he gives a critical appraisal of different approaches in the field of artificial intelligence and law, including the casebased approach; p.12f.). He explicitly moves away from rule-based, case-based and logical approaches to artificial intelligence and law (p. 7f.), and uses a modelling approach instead. This makes it hard to determine whether modelling case-based reasoning using his approach would be closer to rule extraction or to case comparison.

Valente defines cases (as sets of propositions, p. 91, and as descriptions of states of affairs in the world, p. 125), but his cases represent individual behaviour to which legal norms are applied. They do not represent the legal decisions that are central in case-based reasoning. Knowledge about legal decisions would in his ontology fall under different categories of knowledge. Decided cases are for instance sources for legal rules, classificatory rules and conflict resolution. Knowledge about the legal rules extracted from cases would fall under normative knowledge, classificatory knowledge is world knowledge and conflict resolution knowledge is meta-legal knowledge.

Van Kralingen (1995) and Visser (1995) have developed a frame-based conceptual model of the law. They distinguish three types of frames: norm frames, act frames and concept frames. Their approach is explicitly statute-oriented. For instance, their prime example is a model of the Dutch Unemployment Benefits Act. As a result, the role of decided cases is hardly addressed. In their ontology, case-based reasoning would have to be dealt with using the rule-extraction method by modelling the rules extracted from cases in the form of norm frames.

Hage and Verheij (1999) have described the law as a dynamic interconnected system of states of affairs. They use three primitives: states of affairs, events and rules. The primitives are used to analyse legal topics such as classification, rights, proof and validity. Their perspective on the law is rule-based, although Hage and Verheij also discuss principles and goals. In their approach, case-based reasoning would have to be addressed using the rule-extraction method.

Mommers (2002) has built a knowledge-based ontology of the legal domain. He distinguishes entities (e.g., legal norms and decisions), ontological status layers (e.g., existence, validity and recognition), epistemic roles (e.g., reasons and defeaters), relations (e.g., causation, counting as), acts (e.g., applying rules and making decisions) and facts (e.g., brute facts and institutional facts). Mommers recognizes the place of legal decisions (as legal entities) and of making decisions (as acts) in his ontology. He also specifies types of beliefs, with respect to their content (e.g., beliefs based on case law) and origination (e.g., beliefs resulting from interpretation). Mommers does not 
explicitly address case-based reasoning. As a result, it is indeterminate whether applying his ontology would lead to a rule extraction or to a case comparison method towards case-based reasoning.

From the sample of artificial intelligence research on legal ontologies discussed above, it appears that ontological issues concerning cases and case-based reasoning have as yet not been fully addressed in the artificial intelligence community.

\section{Conclusion}

The present paper has shown how case comparison can be used as the basis for modelling case-based reasoning. In the model proposed here (and further elaborated by Roth, 2003), cases are compared in terms of the dialectical arguments expressed in them. The distinction between rule-extraction and case comparison in case-based reasoning leads to different conceptions of cases and thus to different ways of modelling reasoning with them. The distinction has been used to discuss related research on case-based reasoning. A discussion of research on legal ontologies has shown that cases and case-based reasoning have received relatively little attention in such research.

\section{References}

1. Aleven, V. \& Ashley, K.D. (1997). Evaluating a Learning Environment for Case-Based Argumentation Skills. Proceedings of the Sixth International Conference on Artificial Intelligence and Law, pp. 170-179. New York: ACM Press.

2. Aleven, V. (1996). How Different Is Different? Arguing about the Significance of Similarities and Differences. Proceedings of the Third European Workshop: EWCBR-96, pp. 1-15.

3. Aleven, V. (1997). Teaching Case-Based Reasoning Through a Model and Examples. Ph.D. dissertation University of Pittsburgh.

4. Ashley, K.D. (1989). Toward a Computational Theory of Arguing with Precedents: Accomodating Multiple Interpretations of Cases. Proceedings of the Second International Conference on Artificial Intelligence and Law, pp. 93-102. New York: ACM Press.

5. Ashley, K.D. (1990). Modeling Legal Argument: Reasoning with Cases and Hypotheticals. Cambridge: MIT Press/Bradford Books.

6. Ashley, K.D. (1991). Reasoning with cases and hypotheticals in HYPO. International Journal of Man-Machine Studies, Volume 34, pp. 753-796.

7. Bankowski, Z. (1997). Rationales for Precedent. Interpreting Precedents. A comparative study (eds. MacCormick, D.N. and Summers, R.S.). Aldershot: Dartmouth Publishing Company Limited.

8. Bench-Capon, T.J.M. \& Sartor, G. (2001). Theory Based Explanation of Case Law Domains. Proceedings of the Eighth International Conference on Artificial Intelligence and Law, pp. 12-21. New York: ACM Press.

9. Bench-Capon, T.J.M. (1999). Some Observations on Modelling Case Based Reasoning With Formal Argument Models. Proceedings of the Seventh International Conference on Artificial Intelligence and Law, pp. 36-42. New York: ACM Press. 
10. Cross, R. (1977). Precedent in English Law. Third edition. Oxford University Press.

11. Hage, J.C. (1997). Reasoning with Rules. An Essay on Legal Reasoning and Its Underlying Logic. Dordrecht: Kluwer Law and Philosophy Library.

12. Llewellyn, K.N. (1960). The Common Law Tradition. Boston: Little, Brown and Company.

13. Loui, R.P. \& Norman, J. (1995). Rationales and Argument Moves. Artificial Intelligence and Law, Volume 3, pp. 159-189.

14. Loui, R.P. (1997). Eliding the Arguments of Cases. Draft. Downloaded from http://www.cs.wustl.edu/ loui/ratjur.txt.

15. MacCormick, D.N. (1987). Why Cases have Rationes and what these are. In: Precedent in Law (ed. Goldstein, L.), pp. 155-182. Oxford: Clarendon Press.

16. Mommers, L. (2002). Applied legal epistemology. Building a knowledge-based ontology of the legal domain. Dissertation Leiden University.

17. Oliphant, H. (1928). A return to stare decisis. American Bar Association Journal, Volume 14. Reprint in: American Legal Theory (ed. Summers, R.S. 1992), pp. 143-153. Aldershot: Dartmouth Publishing Company Limited.

18. Pollock, J.L. (1987). Defeasible Reasoning. Cognitive Science, Volume 11, pp. 481-518.

19. Pollock, J.L. (1995). Cognitive Carpentry: A Blueprint for How to Build a Person. Cambridge: MIT Press.

20. Prakken, H. \& Sartor, G. (1998). Modelling Reasoning with Precedents in a Formal Dialogue Game. Artificial Intelligence and Law, Volume 6, pp. 231-287.

21. Prakken, H. (2000). An exercise in formalising teleological case-based reasoning. Legal Knowledge and Information Systems: Jurix 2000 (eds. Breuker, J., Leenes, R. and Winkels, R.), pp. 49-57. Amsterdam: IOS Press.

22. Roth, A.C. (2001). The Dialectics of Case Comparison: a Formal Reconstruction. Proceedings of the Fourteenth Annual Jurix Conference (eds. Verheij, B., Lodder, A.R., Loui, R.P. and Muntjewerff, A.J.), 29-40.

23. Roth, A.C. (2003). Case-based reasoning in the law. A formal theory of reasoning by case comparison. Dissertation Universiteit Maastricht.

24. Toulmin, S.E. (1958). The Uses of Argument. London: Cambridge University Press.

25. Valente, A. (1995). Legal Knowledge Engineering. A Modelling Approach. IOS Press, Amsterdam. Dissertation.

26. van Kralingen, R.W. (1995). Frame-based Conceptual Models of Statute Law. Dissertation Leiden University.

27. Verheij, B. (1996). Rules, Reasons, Arguments. Formal studies of argumentation and defeat. Dissertation Universiteit Maastricht.

28. Verheij, B. (1999). Automated Argument Assistance for Lawyers. Proceedings of the Seventh International Conference on Artificial Intelligence and Law, pp. 43-52. New York: ACM Press.

29. Verheij, B. (2000). DefLog - a logic of dialectical justification and defeat. Draft. Downloaded from http://www.metajur.unimaas.nl/ bart/publications.htm.

30. Verheij, B. (2003). DefLog: on the Logical Interpretation of Prima Facie Justified Assumptions. Journal of Logic and Computation, Volume 13, no. 3, pp. 319-346.

31. Visser, P.R.S. \& Bench-Capon, T.J.M. (1998). A Comparison of Four Ontologies for the Design of Legal Knowledge Systems. Artificial Intelligence and Law, Vol. 6, No. 1, pp. $27-$ 57.

32. Visser, P.R.S. \& Winkels, R. (eds.) (1997). Proceedings of the First International Workshop on Legal Ontologies. LEGONT '97. Melbourne: University of Melbourne.

33. Visser, P.R.S. (1995). Knowledge Specification for Multiple Legal Tasks. A Case Study of the Interaction Problem in the Legal Domain. Dissertation Leiden University. 
34. Winkels, R., Van Engers, T. \& Bench-Capon, T.J.M. (eds.) (2001). Proceedings of the Second International Workshop on Legal Ontologies. Amsterdam: Universiteit van Amsterdam. 